\title{
Asymmetry in the Strong-Field Ionization of Rydberg Atoms by Few-Cycle Pulses
}

\author{
A. Gürtler, ${ }^{1}$ F. Robicheaux, ${ }^{2}$ W. J. van der Zande, ${ }^{1,3}$ and L. D. Noordam ${ }^{1}$ \\ ${ }^{1}$ FOM Institute for Atomic and Molecular Physics, Kruislaan 407, 1098SJ Amsterdam, The Netherlands \\ ${ }^{2}$ Department of Physics, Auburn University, Alabama 36849-5311, USA \\ ${ }^{3}$ Department of Molecular and Laser Physics, University of Nijmegen, 6500GL Nijmegen, The Netherlands
}

(Received 29 August 2003; published 22 January 2004)

\begin{abstract}
We present measurements of the electron ejection direction in the ionization of high $(n=90)$ Rydberg states of rubidium subjected to few-cycle radio-frequency (RF) pulses. For weak pulses we find a strong asymmetry for even (cosine) pulses and no asymmetry for odd (sine) pulses. This asymmetry disappears for pulses longer than four RF cycles. For strong pulses, very large asymmetry is found for both sine and cosine pulses that persists up to eight RF cycles and is attributed to initial state depletion effects within a cycle.
\end{abstract}

DOI: 10.1103/PhysRevLett.92.033002

PACS numbers: $32.80 . \mathrm{Rm}$

Nonlinear optical processes in atomic and molecular physics have intrigued researchers ever since highintensity laser sources became available. Notable examples are above-threshold ionization (ATI) [1-3], Coulomb explosion [4], and high-order harmonic generation [5-7]. By definition, these nonlinear processes depend strongly on the oscillating electric field of the driving laser pulse. For the shortest available optical pulses ( $\tau \approx 5 \mathrm{fs}, \lambda=800 \mathrm{~nm}$ ), the pulses contain only a few cycles of the optical field. In this domain, the exact phase between the carrier wave and the envelope becomes important. While the envelope of a short laser pulse can be controlled with high precision nowadays $[8,9]$, the control of the exact electric field, i.e., the carrier-envelope phase, has proven much more challenging and has been accomplished only very recently [10]. The electric field

$$
E_{z}(t)=E_{0} e^{-t^{2} / \tau^{2}} \sin (\omega t+\phi)
$$

for a few-cycle pulse with Gaussian envelope and carrierenvelope (CE) phases of $\phi=0$ (sine pulse) and $\phi=90^{\circ}$ (cosine pulse) is shown in Fig. 1.

In photoionization, the ionized electrons are almost always emitted in equal amounts in the direction of the positive and negative optical fields. Such angular distributions of photoelectrons in ATI experiments have been reported using ground-state atoms exposed to optical pulses [2] as well as Rydberg atoms exposed to manycycle microwave pulses [3].

Paulus and co-workers recently demonstrated by measuring a contingency map that for pulses containing only a few cycles of radiation, the emission direction becomes dependent on the carrier-envelope phase [11]. This dependence was confirmed by Keldish theory [12] and numerical solution of the time-dependent Schrödinger equation for hydrogen in an intense $800 \mathrm{~nm}$ field [13]. At moderate intensities (as used in Ref. [11]), the asymmetry in the ejection direction can be observed only for pulses with $\phi \neq 0^{\circ}$. For cosine pulses $\left(\phi=90^{\circ}\right)$, the positive electric field at $t=0$ is higher than the negative field at any point in time during the short pulse (see Fig. 1). Because of the nonlinearity of the ionization process, the emission in the positive $z$ direction is larger than in the negative $z$ direction. Reference [11] shows clearly that for short pulses ( $6 \mathrm{fs} \hat{=} 2.25$ cycles) an asymmetry is observed, while for longer pulses ( $8 \mathrm{fs} \hat{=} 3$ cycles) no significant asymmetry is found.

In general, how can one understand this asymmetry in terms of dipole transitions? The parts of the wave function created by the absorption of $N, N+2, N+4$, etc., photons, also known as the dressed states, all have the same parity which is opposite to the parity of the dressed states from $N+1, N+3, N+5$, etc., photons. For weak, long laser pulses, these states do not overlap and the asymmetry is absent [Fig. 1(b)]. To observe an asymmetry, it is necessary for the wave function from an odd $N$ to

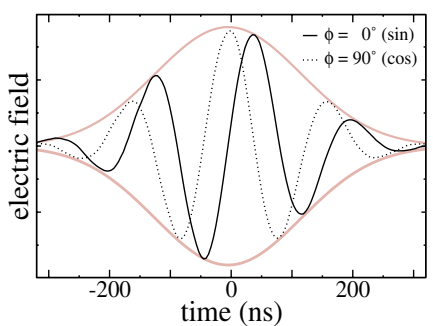

a)

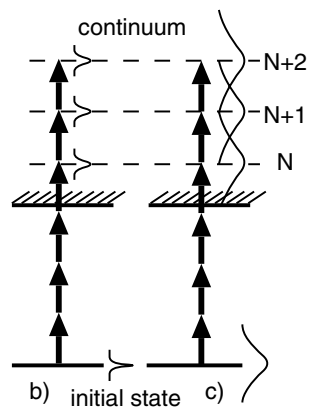

FIG. 1 (color online). (a) Measured radio-frequency pulses with $6 \mathrm{MHz}$ carrier frequency and carrier-envelope phases of $0^{\circ}$ (solid line, sine waveform) and $90^{\circ}$ (dashed line, cosine waveform), respectively. (b) Sketch of a multiphoton ionization process for weak, narrow-band pulses showing the number of photons involved and the width of the resulting electron wave packets in the continuum. Next to the lines representing the initial states, the width of the state is indicated. (c) Same for strong, broadband pulses. Now, the dressed states from $N$ and $N+1$ photons overlap and the interference of these opposite parity states breaks the symmetry of the electron ejection. 
be indistinguishable from an even $N$. This can be accomplished either by the bandwidth of the exciting radiation being large enough that $\omega$ and $2 \omega$ are contained in the bandwidth or by broadening the initial state such that the initial energy gains a width larger than $\omega$. In the case of short (i.e., broadband), strong pulses, the initial state is substantially depleted during a single half-cycle, and both these effects can play a role [Fig. 1(c)]. As a consequence, contributions of opposite parity can interfere constructively in one direction and destructively in the opposite direction [14]. Such interference effects are known to take place in multicolor experiments where frequencies of $\omega$ and $2 \omega$ are mixed and ionization takes place at a frequency of $2 \omega[15,16]$.

In this Letter we studied the asymmetry phenomena in a quite different setting, the ionization of Rydberg atoms by few-cycle radio-frequency (RF) pulses. For low amplitudes we found again that asymmetry appears only for cosine pulses [see Fig. 2(a)]. The asymmetry parameter is defined as

$$
a=\frac{\text { yield }_{\text {right }}-\text { yield }_{\text {left }}}{\text { yield }_{\text {left }}+\text { yield }_{\text {right }}},
$$

which is zero for a symmetric ejection in both directions and \pm 1 for fully directional emission to one side. We define "left" as the direction of the negative field. The relative simplicity of manipulating RF pulses allowed us to repeat the experiment at a large range of pulse durations. We found that for pulses longer than 3.5 cycles the asymmetry is negligible. Surprisingly, we found that for strong pulses of twice the amplitude necessary to deplete

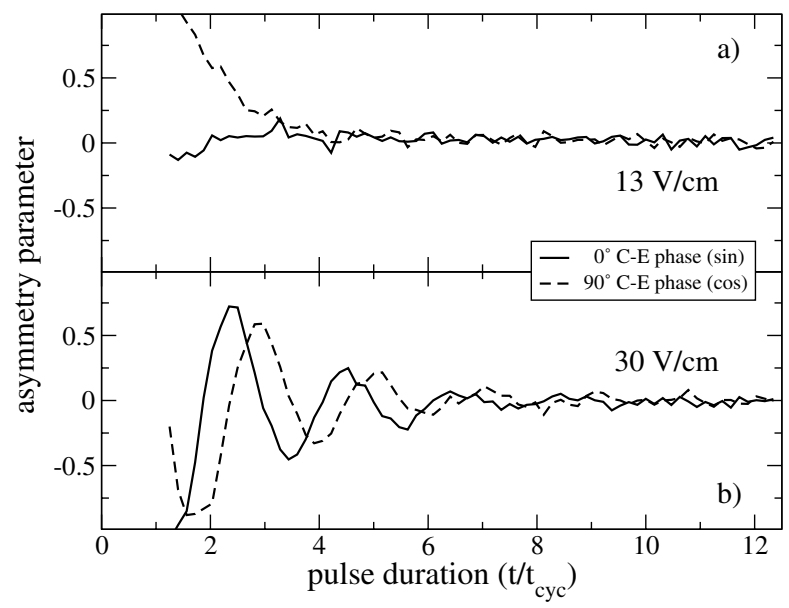

FIG. 2. Left/right asymmetry dependence on the pulse duration in units of the cycle time $t_{\text {cyc }}=80 \mathrm{~ns}$ of a pulse with 12.5 $\mathrm{MHz}$ carrier frequency, $0^{\circ} \mathrm{CE}$ phase (solid line), and $90^{\circ}$ CE phase (dashed line), as well as amplitudes of (a) $13 \mathrm{~V} / \mathrm{cm}$ (35\%-75\% ionization, depending on duration) and (b) $30 \mathrm{~V} / \mathrm{cm}$ RF amplitude (100\% ionization). The ionization process is directional only for few-cycle pulses; for longer pulses the emission becomes symmetric. the Rydberg atom population, the asymmetry in the ejection direction exists for both sine and cosine pulses [see Fig. 2(b)]. The asymmetry is even stronger than for weak pulses and persists up to 8 cycles. For high RF amplitudes the ionization is mostly done by one half-cycle. The effect of increasing the pulse duration while keeping the envelope amplitude constant in this case is to enable an earlier half-cycle to ionize most of the Rydberg population such that the ejection direction reverses. For very long pulses, the ionization is spread over many half-cycles on the rising edge of the pulse and the emission becomes symmetric. The sine and cosine traces in Fig. 2(b) are shifted with respect to each other by $T / 2$, with $T$ being the RF cycle time. A detailed calculation showing the interplay among CE phase, amplitude, and pulse duration which results in this $T / 2$ shift is beyond the scope of this Letter.

Prior to describing the experimental setup we discuss the mechanisms of optical and RF ionization. In multiphoton ionization of ground-state atoms, high-intensity laser pulses produce fields $\gtrsim 10^{8} \mathrm{~V} / \mathrm{cm}$ which suppress the binding Coulomb potential sufficiently to allow the atom to ionize by tunneling or above-barrier ionization. In contrast, for highly excited Rydberg atoms the quasistatic fields needed to ionize the atom by the same mechanism are on the order of a few $\mathrm{V} / \mathrm{cm}$. The orbit time of the Rydberg electron is about 6 orders of magnitude longer than that of a ground-state electron. In both systems the electron samples the potential much faster than the time variation of the field. The combined dynamics of the atomic system and the time-varying field of a $n=90$ Rydberg state subjected to a $10 \mathrm{~V} / \mathrm{cm}$ radiofrequency pulse and a ground-state atom subjected to a $10^{8} \mathrm{~V} / \mathrm{cm}$ optical pulse are therefore comparable [17]. An advantageous aspect of experiments at RF frequencies is the continuous control of all pulse parameters.

RF pulses are easily synthesized in arbitrary waveforms. For the experiments presented here, we used an Agilent 33250A arbitrary pulse form generator to produce pulse forms with a Gaussian envelope and carrier frequencies of up to $25 \mathrm{MHz}$ with controllable $\mathrm{CE}$ phases as shown in Fig. 1. The generated pulse forms are then amplified by a RF amplifier to amplitudes of up to $25 \mathrm{~V}$. In the experimental setup we excite $\mathrm{Rb}$ atoms from a resistively heated oven to a Rydberg state around $n=$ 90 using a $10 \mathrm{~Hz}$ narrow-band $\left(0.13 \mathrm{~cm}^{-1}\right)$ nanosecond dye laser. The excitation takes place between two capacitor plates spaced by $4.65 \mathrm{~mm}$ which are set up in a $50 \Omega$ transmission line configuration for the radio frequency. The RF pulse is applied to one of the plates $200 \mathrm{~ns}$ after excitation. Electrons ionized by the negative part of the $\mathrm{RF}$ are pushed through a grid-covered hole in the second plate by the RF field. The electron yield is detected with a microchannel plate detector with subcycle time resolution. The emission in the other direction is measured by repeating this procedure with the inverted pulse form, such that now electrons ionized by the positive part of the 
first pulse are detected. In the time from excitation to ionization of the Rydberg atoms, the initially excited $90 \mathrm{~d}$ state evolves through processes like precession in the earth magnetic field into states with higher angular momenta and magnetic quantum numbers. The resulting mixture of states displays a broad threshold for electric field ionization and ionizes in bursts upon application of a RF field [17].

In Fig. 3 we show the influence of the RF amplitude on the symmetry in the ejection of the ionized electrons. We plot the ionization yield for a $90 d$ Rydberg state subjected to a $300 \mathrm{~ns}$ RF pulse with a Gaussian envelope and $6 \mathrm{MHz}$ carrier frequency dependent on the RF amplitude. The carrier-envelope phase is set to $45^{\circ}$. The dotted and dashed lines represent the electron yield ejected in left and right directions, respectively, and the solid line represents the total ionization yield. The curves can be roughly divided into three sections: for very low amplitudes, the electron flux is spread evenly over both directions. The ionized fraction is negligible, such that each subsequent half-cycle finds the same situation and ionizes a certain fraction. For the $45^{\circ}$ pulse shape, two half-cycles contribute to the ejection in each direction and in this situation this results in symmetric ejection. For amplitudes higher than $\approx 11 \mathrm{~V} / \mathrm{cm}$ a slight asymmetry can be seen. In this region, more than $10 \%$ of the Rydberg population is ionized by the RF pulse, such that the order in which the half-cycles follow upon each other becomes important. Finally, for RF amplitudes exceeding $20 \mathrm{~V} / \mathrm{cm}$, the Rydberg population is depleted at the end of the pulse. In this region, a considerable fraction of the population is ionized by a single half-cycle and the emission becomes directional. For even higher amplitudes, an earlier half-cycle takes over and the emission direction is reversed.

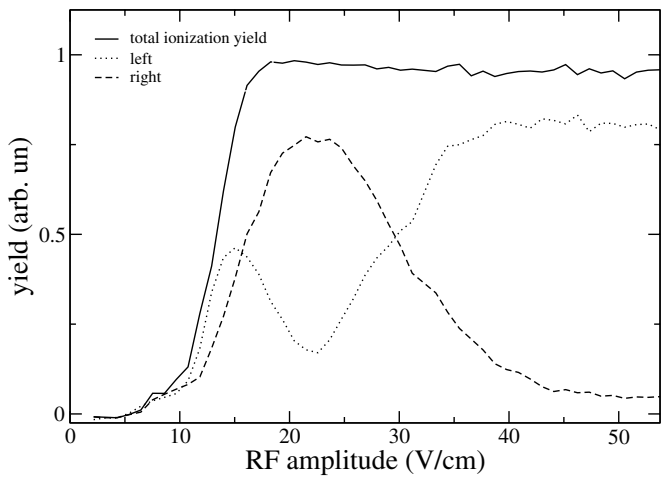

FIG. 3. Ionization yield ejected to the left (dotted line) and to the right (dashed line) for a $90 \mathrm{~d}$ initial state subjected to a $6 \mathrm{MHz}, 300 \mathrm{~ns}$ pulse with a carrier-envelope phase of $45^{\circ}$. The solid line shows the total ionization yield. For low ionized fractions, the angular distribution is uniform. In the strong-field regime, where the Rydberg population gets more and more depleted, the ejection direction gets more and more dependent on the amplitude.
Figure 4 shows the asymmetry parameter $a$ depending on the CE phase for RF amplitudes of $13 \mathrm{~V} / \mathrm{cm}$ (dashed line), $23 \mathrm{~V} / \mathrm{cm}$ (dotted line), and $30 \mathrm{~V} / \mathrm{cm}$ (solid line) and a pulse containing about two cycles at full width at half maximum. It is apparent that the curves are very different. Comparison with Fig. 3 shows that the initial Rydberg population is depleted after the pulse for amplitudes $\gtrsim 18 \mathrm{~V} / \mathrm{cm}$. Only for low RF amplitudes (dashed line) the asymmetry behaves as expected from the RF pulse shape, i.e., symmetric emission for $\phi \approx 0,180^{\circ}$ (compare Fig. 1) and an asymmetric emission for $\phi \approx$ $90^{\circ}$. The observed shift of $10^{\circ}$ to higher values can be attributed to stray electric fields caused, e.g., by contact potentials of $\mathrm{Rb}$ deposited on the plates and slight asymmetries in the RF pulse shapes. For high RF amplitudes (dotted and solid lines), the ionization process is easily dominated by a few or even a single half-cycle of the RF field. Therefore the ejection becomes highly directional, depending on the exact pulse shape. If we follow the solid line in Fig. 4 (highest field), the emission is dominated by a single half-cycle in the left direction just before the peak of the envelope at $\phi=0$. With a growing CE phase, this half-cycle ionizes less and less and a second, later half-cycle with opposite polarity begins to contribute until both of them contribute equally to the ionization signal at $\phi \approx 53^{\circ}$. The influence of the first half-cycle further decreases and a third half-cycle again in the left direction starts to contribute at $\phi \approx 140^{\circ}$. For even higher $\mathrm{CE}$ phases, the influence of the third half-cycle grows. This shows that for high amplitudes the exact behavior is complex and depends on the details of the underlying strong-field ionization mechanisms. This requires detailed theoretical investigations.

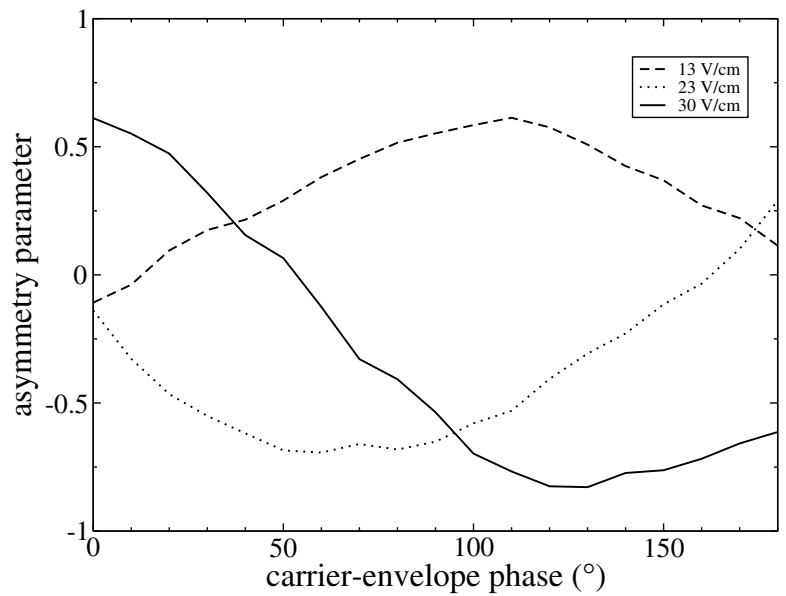

FIG. 4. Dependence of the ejection direction on the carrierenvelope phase. Shown is the ejection asymmetry parameter for RF amplitudes of $13 \mathrm{~V} / \mathrm{cm}$ (dashed line), $23 \mathrm{~V} / \mathrm{cm}$ (dotted line), and $30 \mathrm{~V} / \mathrm{cm}$ (solid line). A value of zero means symmetric ejection in both directions. The Rydberg population is depleted for amplitudes $\gtrsim 18 \mathrm{~V} / \mathrm{cm}$. The $6 \mathrm{MHz}$ pulses are 300 ns long. 
The experiments presented here suggest a direction for further experiments in the optical domain. For very strong fields the oscillatory behavior seen in Fig. 2(b) should be present in the optical case as well. The pulse energy of advanced CE phase stabilized laser pulses is not easily increased to arbitrary values to deplete the ground state of the widely used noble gas atoms. Using an alkali metal such as rubidium or cesium with a much lower ionization potential could allow for this kind of experiments even at today's pulse energies.

It has been suggested that the asymmetry in ionization could be used to determine the CE phase of a fewcycle pulse [11]. The results at RF frequencies shown in Fig. 4 do not display an unambiguous relationship between the asymmetry and the phase, indicating that the asymmetry should be measured for at least two intensities in the strong-field regime to deduce the CE phase from the result.

The RF domain is very suitable to explore phenomena dependent on the phases between two frequencies and the carrier-envelope phase of an ultrashort two-color pulse. Experiments in this direction are currently being performed and might lead to further suggestions for experiments in the optical domain.

In conclusion, we measured the ejection direction of Rydberg electrons ionized by radio-frequency pulses depending on amplitude, duration, and the carrier-envelope phase of the pulses. We found that the ejection direction for low RF amplitudes strongly depends on the CE phase for short pulses. For high amplitudes, the ejection direction is always asymmetric and strongly depends on the exact field for few-cycle pulses. For many-cycle pulses the electron emission becomes symmetric in both cases.

The authors thank Marc Vrakking for valuable discussions and Huib Bakker for careful reading of the manuscript. A. G. acknowledges financial support from the European Community's Human Potential Programme under Contract No. HPRN-CT-1999-00129, COCOMO.
F. R. was supported by the NSF. This work is part of the research program of the "Stichting voor Fundamenteel Onderzoek der Materie (FOM)," which is financially supported by the "Nederlandse Organisatie voor Wetenschappelijk Onderzoek (NWO)."

[1] P. Agostini, F. Fabre, G. Mainfray, G. Petite, and N. K. Rahman, Phys. Rev. Lett. 42, 1127 (1979).

[2] V. Schyja, T. Lang, and H. Helm, Phys. Rev. A 57, 3692 (1998).

[3] T. F. Gallagher, Phys. Rev. Lett. 61, 2304 (1988).

[4] J. H. Posthumus, A. J. Giles, M. R. Thompson, and K. Codling, J. Phys. B 29, 5811 (1996).

[5] B. Sheehy, J. D. D. Martin, L. F. DiMauro, P. Agostini, K. J. Schafer, M. B. Gaarde, and K. C. Kulander, Phys. Rev. Lett. 83, 5270 (1999).

[6] J. Zhou, J. Peatross, M. M. Murnane, H. C. Kapteyn, and I. P. Christov, Phys. Rev. Lett. 76, 752 (1996).

[7] A. LHuillier and P. Balcou, Phys. Rev. Lett. 70, 774 (1993).

[8] A. M. Weiner, Rev. Sci. Instrum. 71, 1929 (2000).

[9] T. Brixner, A. Oehrlein, M. Strehle, and G. Gerber, Appl. Phys. B 70, S119 (2000).

[10] A. Baltuška et al., Nature (London) 421, 611 (2003).

[11] G. G. Paulus, F. Grasbon, H. Walther, P. Villoresi, M. Nisoli, S. Stagira, E. Priori, and S. de Silvestri, Nature (London) 414, 182 (2001).

[12] D. B. Milošević, G. G. Paulus, and W. Becker, Phys. Rev. Lett. 89, 153001 (2002).

[13] S. Chelkowski and A. D. Bandrauk, Phys. Rev. A 65, 061802 (2002).

[14] Note that in optical experiments such as in Ref. [11] $N \sim$ 10 , while in the RF experiments presented here $N \sim 10^{4}$.

[15] D.W. Schumacher, F. Weihe, H. G. Muller, and P. H. Bucksbaum, Phys. Rev. Lett. 73, 1344 (1994).

[16] K. J. Schafer and K. C. Kulander, Phys. Rev. A 45, 8026 (1992).

[17] A. Gürtler, M. J. J. Vrakking, W. J. van der Zande, and L. D. Noordam (to be published). 